\title{
Modulation of proliferation and differentiation of gingiva-derived mesenchymal stem cells by concentrated growth factors: Potential implications in tissue engineering for dental regeneration and repair
}

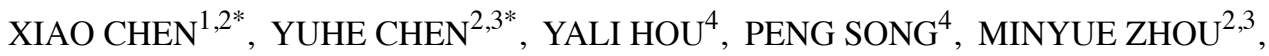 \\ MINHAI NIE ${ }^{2,3}$ and XUQIAN LIU ${ }^{2,3}$ \\ ${ }^{1}$ Department of Orthodontics, Mianyang Stomatological Hospital, Mianyang, Sichuan 621000; \\ ${ }^{2}$ Laboratory of Oral and Maxillofacial Reconstruction and Regeneration, Southwest Medical University; \\ ${ }^{3}$ Department of Periodontics and Oral Mucosa, Affiliated Stomatology Hospital, \\ Southwest Medical University, Luzhou, Sichuan 646000; ${ }^{4}$ Department of Oral Pathology, \\ College and Hospital of Stomatology, Hebei Medical University, Shijiazhuang, Hebei 050000, P.R. China
}

Received November 17, 2018; Accepted April 10, 2019

DOI: $10.3892 /$ ijmm.2019.4172

\begin{abstract}
The aim of the present study was to evaluate the proliferation and osteogenic differentiation ability of gingiva-derived mesenchymal stem cells (GMSCs) cultured with different concentrations of concentrated growth factors (CGF). GMSCs were isolated from gingival connective tissues and characterized by flow cytometry, immunofluorescence staining and immunohistochemical staining. Cell proliferation activity was determined by the MTT assay, and the effect of CGF on MCSCs was detected with the Cell Counting Kit (CCK)-8 assay.Mineralization induction was evaluated by alkaline phosphatase (ALP)-positive cell staining and mineralized nodule formation assay. Dentin matrix acidic phosphoprotein (DMP)1, dentin sialophosphoprotein (DSPP), bone morphogenetic protein (BMP)2 and runt-related transcription factor (RUNX)2 mRNA and protein expression were evaluated by reverse transcription-quantitative polymerase chain reaction (RT-qPCR) analysis and western blotting. The flow cytometry, immunofluorescence staining and immunohistochemical staining results indicated that the cultured cells were GMSCs. The MTT assay results revealed that the third-generation
\end{abstract}

Correspondence to: Professor Xuqian Liu or Professor Minhai Nie, Department of Periodontics and Oral Mucosa, Affiliated Stomatology Hospital, Southwest Medical University, 2 Jiangyang South Street, Luzhou, Sichuan 646000, P.R. China

E-mail: liuxuqianwork@163.com

E-mail: nieminhai@126.com

${ }^{*}$ Contributed equally

Key words: gingiva-derived mesenchymal stem cells, concentrated growth factors, gingiva, osteogenesis, mineralization gingival stem cells exhibited the highest proliferative capacity, and the CCK- 8 results indicated that 10\% CGF achieved the most prominent promotion of GMSC proliferation. ALP activity analysis and mineralized nodule assay demonstrated that CGF may successfully induce osteogenic differentiation of GMSCs, whereas RT-qPCR and western blot analyses demonstrated that CGF is involved in the differentiation of GMSCs by regulating the expression of DMP1, DSPP, BMP2 and RUNX2 $(\mathrm{P}<0.05)$. In conclusion, CGF were demonstrated to promote the proliferation and osteogenic differentiation of GMSCs. Therefore, CGF may be applied in tissue engineering for tooth regeneration and repair.

\section{Introduction}

Mesenchymal stem cells (MSCs) were first identified in the bone marrow, but can also be obtained from various tissues, such as fat, synovial membrane, muscle, skin, trabecular bone, articular cartilage, umbilical cord and placenta, and they may differentiate into osteoblasts, chondroblasts, myoblasts, adipoblasts, myocardium and skin $(1,2)$. Due to their multiple differentiation potential, bone marrow MSCs (BMSCs) have played a key role in cell-based therapy and tissue engineering in recent years. However, limitations associated with cell isolation, aging and limited proliferative capacity have restricted the potential clinical applicability of BMSCs $(3,4)$. Gingiva-derived MSCs (GMSCs) are a novel type of pluripotent MSCs that exhibit self-renewal, multipotent differentiation potential and immunomodulatory capacities (4-6). As novel postnatal stem cells, GMSCs have been attracting increasing attention due to their easy isolation, high proliferative capacity, homogeneity, stable phenotype and, notably, the fact that they maintain a normal karyotype and telomerase activity during prolonged culture (4). Therefore, GMSCs are considered to be an optimal candidate cell resource for tissue engineering and cell-based therapies. 
Concentrated growth factors (CGF), initially identified by Sacco (unpublished data), represent a novel generation of platelet concentrate products $(3,7)$. CGF are produced by centrifuging venous blood using a specialized centrifugation procedure. The alternated and controlled speed centrifugation permits the isolation of a larger and denser fibrin matrix (8), which forms richer layers of growth factors and produces an enriched fibrin clot (9). This fibrin clot exhibits high cohesion due to the agglutination of fibrinogen, factor XIII and thrombin factor XIIIa, and may provide protection from plasmin degradation, resulting in higher fibrin tensile strength and stability (10). A number of previous studies have demonstrated that CGF can promote, improve and enhance tissue repair and regeneration $(7,11,12)$. However, those studies were largely clinical and the underlying mechanism has not yet been explored in depth. The aim of the present study was to investigate the effect of different concentrations of CGF on GMSC proliferation and osteogenic differentiation.

\section{Materials and methods}

Isolation and culture of GMSCs. In the present study, samples were harvested from the normal gingival tissue of patients undergoing crown lengthening surgery. The study protocol was approved by the Ethics Committee of the Affiliated Stomatological Hospital of Southwest Medical University and the patients provided written informed consent prior to tissue collection. The gingival tissues were washed three times with PBS containing $400 \mu \mathrm{g} / \mathrm{ml}$ streptomycin and $400 \mathrm{U} / \mathrm{ml}$ penicillin, the epithelial layer was separated from connective tissue and the connective tissue was minced into $0.5-\mathrm{mm}^{3}$ pieces. The tissue explants were then placed into Dulbecco's modified Eagle's medium (DMEM; HyClone; GE Healthcare Life Sciences) supplemented with $15 \%$ fetal bovine serum (Hangzhou Sijiqing Biological Engineering Materials Co., Ltd.) and incubated at $37^{\circ} \mathrm{C}$ in a humidified atmosphere of $5 \% \mathrm{CO}_{2}$. Cells were subcultured to $80 \%$ confluence with $0.25 \%$ trypsin/EDTA solution

Flow cytometry for surface marker analysis. GMSCs were prepared as single-cell suspensions via trypsinization and resuspended in blocking buffer containing Hank's balanced salt solution supplemented with $1 \%$ BSA for $30 \mathrm{~min}$. Approximately $1 \times 10^{6}$ cells/ml were incubated with primary antibodies against CD90 (mouse anti-human monoclonal antibody, H30901-09G, Tianjin Sungene Biotech Co., Ltd.), CD105 (mouse anti-human monoclonal antibody, H31051-09H, Tianjin Sungene Biotech Co., Ltd.), CD73 (mouse anti-human monoclonal antibody, 85-11-0739-41; eBioscience) and CD45 (mouse anti-human monoclonal antibody, H20451-09G, Tianjin Sungene Biotech Co., Ltd.) for $30 \mathrm{~min}$ at $4^{\circ} \mathrm{C}$ in the dark. After washing three times with PBS, the cells were fixed in fluorescence-activated cell sorting fix solution and then analyzed using a Beckman Coulter flow cytometer and FACScan Cytomics (Becton, Dickinson and Company).

Immunofluorescence analysis. Following fixation with $4 \%$ paraformaldehyde, the GMSCs were permeabilized with methanol and blocked with 5\% BSA in PBS for $20 \mathrm{~min}$. Subsequently, the GMSCs were immunolabeled with anti- bodies against CD90 (rabbit anti-human monoclonal antibody, 1:100, EPR313, Abcam) S100A4 (rabbit anti-human monoclonal antibody, 1:200, EPR2761(2), Abcam), vimentin (rabbit anti-human monoclonal antibody, 1:100, EPR3776, Abcam) and cytokeratin (mouse anti-human monoclonal antibody, 1:100, AM10031PU-S, OriGene Technologies, Inc.). Following incubation, the cells were washed with PBS, incubated with fluorescein isothiocyanate-conjugated secondary antibody and counterstained with DAPI. The samples were observed under a fluorescence microscope (Olympus Corporation).

Immunohistochemical analysis. Briefly, endogenous peroxidase activity within the sections was quenched by incubating the sections with $3 \% \mathrm{H}_{2} \mathrm{O}_{2}$ for 10 min following dewaxing and hydration. GMSCs were incubated with primary antibodies against vimentin (rabbit anti-human monoclonal antibody, 1:100, EPR3776, Abcam), CD90 (rabbit anti-human monoclonal antibody, 1:100, EPR313, Abcam), CD73 (rabbit anti-human monoclonal antibody, 1:200, EPR6114, Abcam) and cytokeratin (mouse anti-human monoclonal antibody, 1:100, AM10031PU-S, OriGene Technologies, Inc.) for $30 \mathrm{~min}$ at room temperature, followed by incubation with a secondary antibody. In negative controls, the primary antibody was replaced with PBS. The sections were then counterstained with hematoxylin.

Cell viability analysis. Cell viability was evaluated using the MTT assay (13). GMSCs were incubated with MTT in culture medium at $37^{\circ} \mathrm{C}$ for $4 \mathrm{~h}$. The medium was then aspirated from the well, and $150 \mu 1$ dimethyl sulfoxide (Hebei Bio-High Technology Development Co.) was added to each well. The plates were agitated on a plate shaker for $20 \mathrm{~min}$, and $150 \mathrm{ml}$ of this solution was transferred to a 96-well plate (Costar; Corning, Inc.) using opaque-walled transparent-bottomed plates. The optical density was read at $570-650 \mathrm{~nm}$ on a plate reader, and data are expressed as absorbance.

Cell activity at different concentrations of CGF. Passage 3 GMSCs were prepared into a cell suspension and inoculated in 96-well plates at a density of $1 \times 10^{5}$ cells/well. After $24 \mathrm{~h}$ of culture, DMEM was replaced with 5, 10, 20 and 40\% CGF at $24,48,72$ and $92 \mathrm{~h}$ in the same conditioning culture. All treated cells were assessed via CCK-8 assay.

Osteogenic differentiation. Passage 3 GMSCs were seeded in a 24-well plate (Costar; Corning, Inc.) at a density of $2 \times 10^{4}$ cells/well and incubated overnight. The following day, cells were divided into three groups as follows: i) The control group, cultured with DMEM; ii) the pure mineralization group, cultured with osteogenesis induction medium; and iii) the experimental group, cultured with osteogenesis induction medium $+10 \%$ CGF. On days 7, 14 and 21, Alizarin Red S staining (Sigma-Aldrich; Merck KGaA) was performed to observe the formation of mineralized nodules.

Alkaline phosphatase (ALP) enzyme activity assay. ALP activity was assessed to determine ALP expression of GMSCs cultured under different conditions. GMSCs were cultured in the three culture media detailed above and the ALP activity of GMSCs was detected using an ALP assay kit (Thermo Fisher 
Table I. Primers used for qPCR analysis.

\begin{tabular}{lll}
\hline Gene & \multicolumn{1}{c}{ Forward (5'-3') } & \multicolumn{1}{c}{ Reverse (3'-5') } \\
\hline DSPP & GGCGATGCAGGTCACAATGA & GTGCCTGTGTTACCTCAGC \\
DMP1 & TCAGGAAGAGGTGGTGAGTGAGTC & ACTGGATTCGCTGTCTGCTTGC \\
BMP2 & TGACGAGGTCCTGAGCGAGTTC & TGAGTGCCTGCGATACAGGTCTAG \\
RUNX2 & AACAGCAGCAGCAGCAGCAG & GCACCGAGCACAGGAAGTTGG \\
GAPDH & GGTGAAGGTCGGTGTGAACG & CTCGCTCCTGGAAGATGGTG \\
\hline
\end{tabular}

qPCR, quantitative polymerase chain reaction; DSPP, dentin sialophosphoprotein; DMP1, dentin matrix acidic phosphoprotein 1; BMP2, bone morphogenetic protein 2; RUNX2, runt-related transcription factor 2; GAPDH, glyceraldehyde-3-phosphate dehydrogenase.
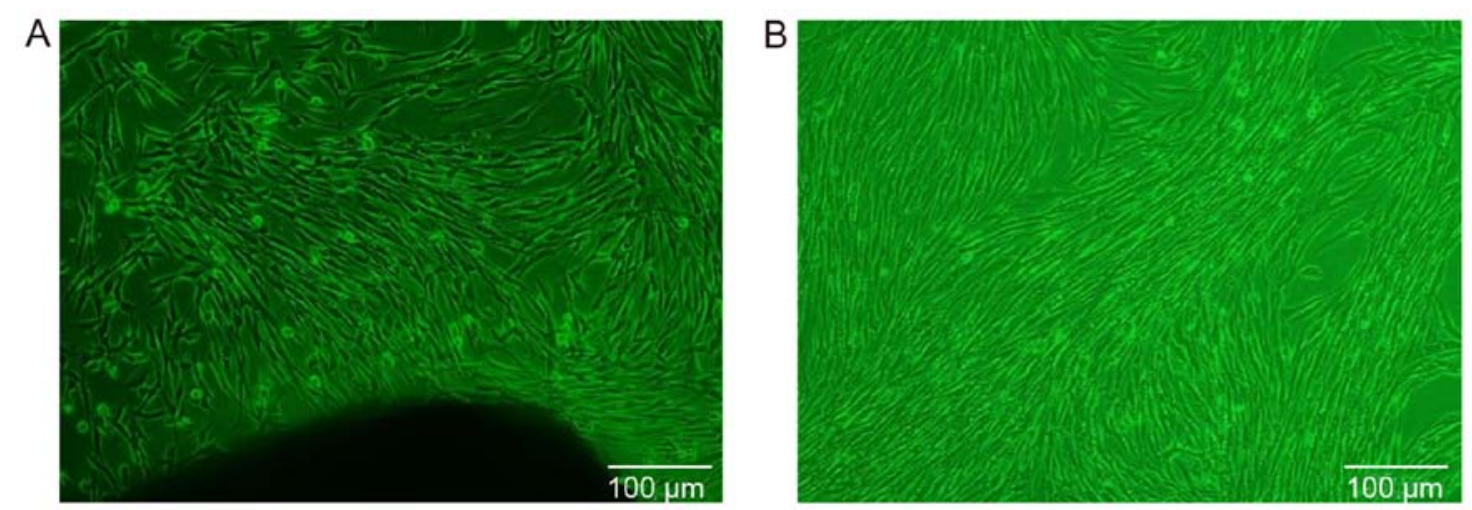

Figure 1. Culture of gingiva-derived mesenchymal stem cells in vitro (magnification, x100). (A) Primary culture: The cells exhibited a long fusiform or polygonal shape. (B) Subculture: The cells exhibited consistent morphology, including fusiform shape with a plump cell body, clear nucleus and fibroblast-like phenotype.

Scientific, Inc.). The results were measured at $405 \mathrm{~nm}$ in a spectrophotometer using a microplate reader (Sunrise; Tecan Group, Ltd.).

Reverse transcription-quantitative polymerase chain reaction (RT-qPCR) analysis. Cells in all three groups were harvested and RNA was extracted using TRIzol reagent (Qiagen $\mathrm{GmbH}$ ) according to the manufacturer's protocol. RT to cDNA was then performed using a Sensiscript RT kit (Thermo Fisher Scientific, Inc.), followed by qPCR. The thermocycling conditions were as follows: $95^{\circ} \mathrm{C}$ for $10 \mathrm{~min} ; 40$ cycles at $95^{\circ} \mathrm{C}$ for $20 \mathrm{sec}, 56^{\circ} \mathrm{C}$ for $30 \mathrm{sec}$ and extension at $72^{\circ} \mathrm{C}$ for $31 \mathrm{sec}$. The relative quantities of mRNA were calculated using the $2^{-\triangle \Delta C q}$ method (14) and normalized to the housekeeping gene GAPDH. The PCR primer sequences are listed in Table I.

Western blot analysis. Cells were lysed with buffer containing $50 \mathrm{mM}$ Tris- $\mathrm{HCl}$ (pH 7.5), $5 \mathrm{mM}$ EDTA, $150 \mathrm{mM} \mathrm{NaCl}, 0.5 \%$ Triton X-100, $10 \mathrm{mM}$ sodium fluoride, $20 \mathrm{mM}$ 2-ME, $250 \mu \mathrm{M}$ sodium orthovanadate and $1 \mathrm{mM}$ phenylmethane sulfonyl fluoride, and incubated at $4^{\circ} \mathrm{C}$ for $1 \mathrm{~h}$. The cell lysates were ultrasonicated and centrifuged at $12,000 \mathrm{x} \mathrm{g}$ for $10 \mathrm{~min}$. Protein concentrations were determined with the bicinchoninic acid assay. Protein samples were separated by $8 \%$ SDS-PAGE and electroblotted onto nitrocellulose membranes (EMD Millipore). Following blocking with TBS and 5\% non-fat dry milk for $2 \mathrm{~h}$, the membrane was incubated overnight at $4^{\circ} \mathrm{C}$ with antibodies against dentin sialophosphoprotein (DSPP, rabbit anti-human polyclonal antibody, 1:1,000, bs-10316R, Bioss), bone morphogenetic protein (BMP)2 (rabbit anti-human polyclonal antibody, 1:1,000, bs-1012R, Bioss), dentin matrix acidic phosphoprotein 1 (DMP1, rabbit anti-human polyclonal antibody, 1:1,000, bs-12359R, Bioss) and runt-related transcription factor (RUNX)2 (rabbit anti-human polyclonal antibody, 1:1,000, bs-1134R, Bioss), followed by incubation with a horseradish peroxidase-conjugated secondary antibody for $45 \mathrm{~min}$ at room temperature. After each incubation, the membrane was thoroughly washed with TBS-Tween-20. Subsequently, a coloring reaction was performed with ECL and the ratio was quantified using a GelDoc XR System (Bio-Rad Laboratories, Inc.).

Statistical analysis. All data were analyzed with SPSS 19.0 (IBM Corp.) and are expressed as mean \pm standard deviation. Statistical analysis was performed using one-way ANOVA followed by a SNK-q post hoc test. $\mathrm{P}<0.05$ was considered to indicate a statistically significant difference.

\section{Results}

Cytoskeletal morphology of GMSCs. The first adherent cells appeared 3-14 days after initiation of the primary culture, at which time the cells displayed a long fusiform or polygonal shape (Fig. 1A). When the primary cells reached $80 \%$ confluence, cell subculture was conducted. Following cell subculture, the cells exhibited consistent morphology, including a fusiform shape with a plump cell body, a clear 

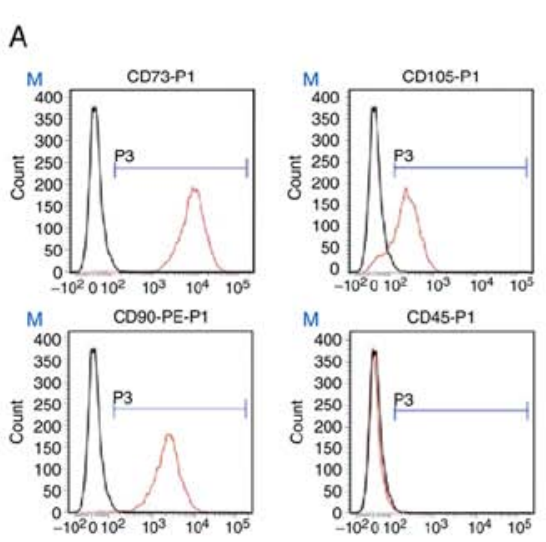

B

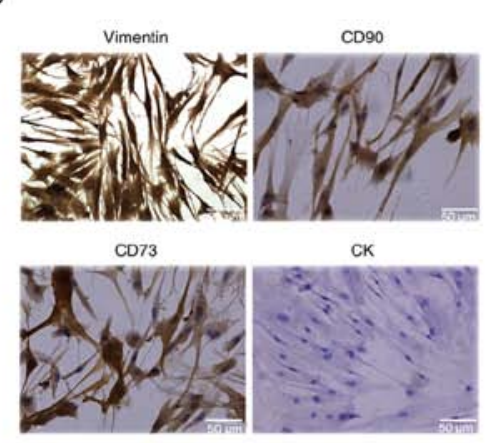

C

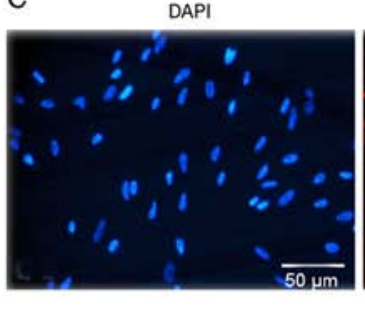

DAPI

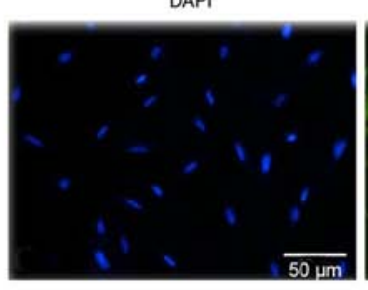

DAPI

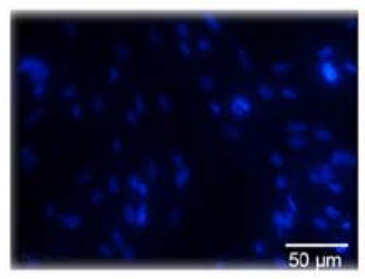

DAPI

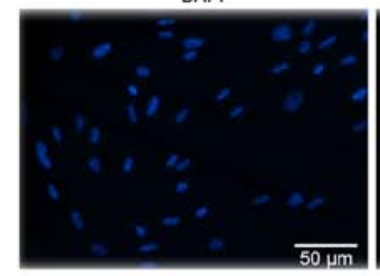

$\operatorname{CD} 90$

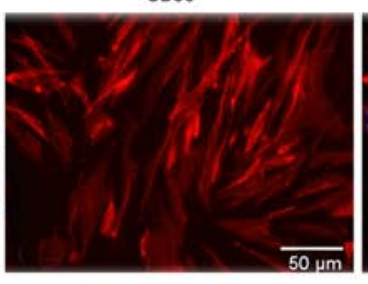

S100A4

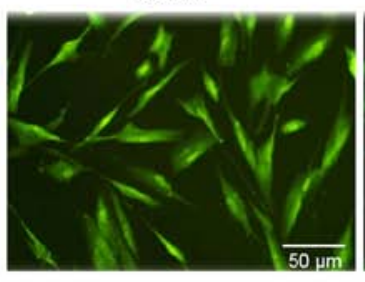

Vimentin

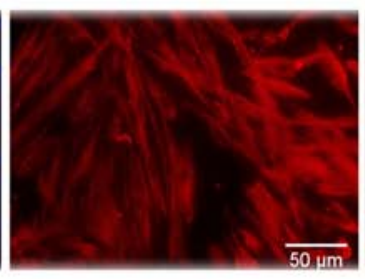

CK

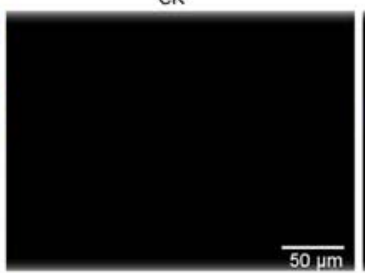

CD90/DAPI

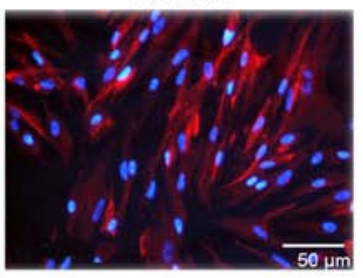

S100A4/DAPI

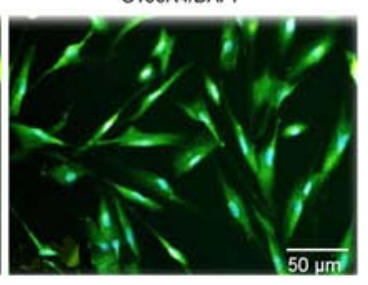

Vimentin'DAPI

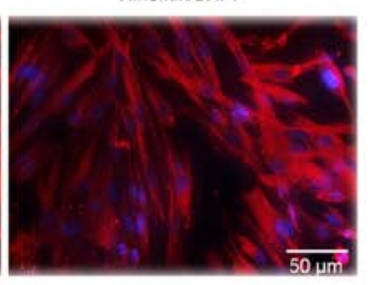

CKIDAPI

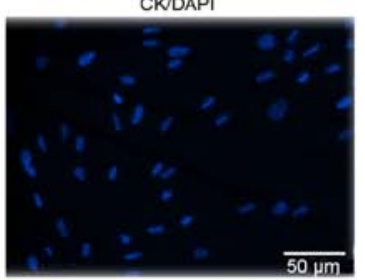

Figure 2. Characterization of GMSCs. (A) Flow cytometry analysis indicated that human GMSCs expressed CD73, CD105 and CD90, but not CD45. (B) Expression of stem cell markers in GMSCs. Cells cultured in a 24-well plate were fixed and stained with specific antibodies against human vimentin, CD90, CD73 and cytokeratin (CK). The cells were incubated with rhodamine- or fluorescein isothiocyanate-conjugated secondary antibodies and then observed under a fluorescence microscope (magnification, x200). (C) Immunofluorescence staining revealed that the cytoplasm of CD90- and vimentin-stained cells exhibited red fluorescence, and the cytoplasm of S100A4-stained cells exhibited green fluorescence. The nuclei of all cells exhibited blue fluorescence, whereas the cytokeratin-stained cells exhibited no fluorescence (magnification, x200). GMSCs, gingiva-derived mesenchymal stem cells.

nucleus and a fibroblast-like phenotype. When the cells were densely clustered, they were arranged in a circinate or radial pattern (Fig. 1B).

Characterization of GMSCs. Flow cytometric analysis revealed that GMSCs were uniformly positive for CD73, CD105 and CD90, but did not express the hematopoietic stem cell marker CD45. The positive cell count was $99.83 \%$ for CD73, $99.74 \%$ for CD90, 82.35\% for CD105 and 2.05\% for CD45. These findings suggested that the cultured cells derived from gingival tissue had the biological characteristics of MSCs (Fig. 2A).

To further identify the GMSCs, immunohistochemical and immunofluorescence staining were conducted. Immunohistochemical peroxidase stained the cytoplasm brown and the nuclei blueish-purple for CD73, CD90 and vimentin, whereas on cytokeratin staining both the cytoplasm and nucleus remained blueish-purple (Fig. 2B). Notably, similar results were obtained by DAPI staining. The cytoplasm of CD90- and vimentin-stained cells exhibited red fluorescence, and the cytoplasm of S100A4-stained cells exhibited green fluorescence. The nuclei of all cells exhibited blue fluores- cence, except the cells stained positive for cytokeratin, which exhibited no fluorescence in the cytoplasm. These results indicated that the cultured cells were stem cells of mesenchymal origin, and were determined to be GMSCs (Fig. 2C).

Proliferative activity of GMSCs. In order to determine the optimal proliferative capacity of GMSCs, the MTT assay was performed to plot the growth curve of each generation of GMSCs. The results of the MTT assay indicated that the GMSCs exhibited logarithmic growth on days 3-4 following inoculation, and the cell number tended to stabilize on days 7 and 8 . The cell growth curve had a typical ' $S$ ' shape, and it displayed three growth stages: Latency, logarithmic growth period and plateau. The line chart demonstrated that secondand third-generation cells exhibited a strong proliferation capacity, whereas fourth- and fifth-generation cells exhibited a weaker capacity (Fig. 3). Therefore, third-generation cells were used in the following experiments.

CGF promote the proliferation of GMSCs. CGF can promote, improve and enhance tissue repair and regeneration. Following 


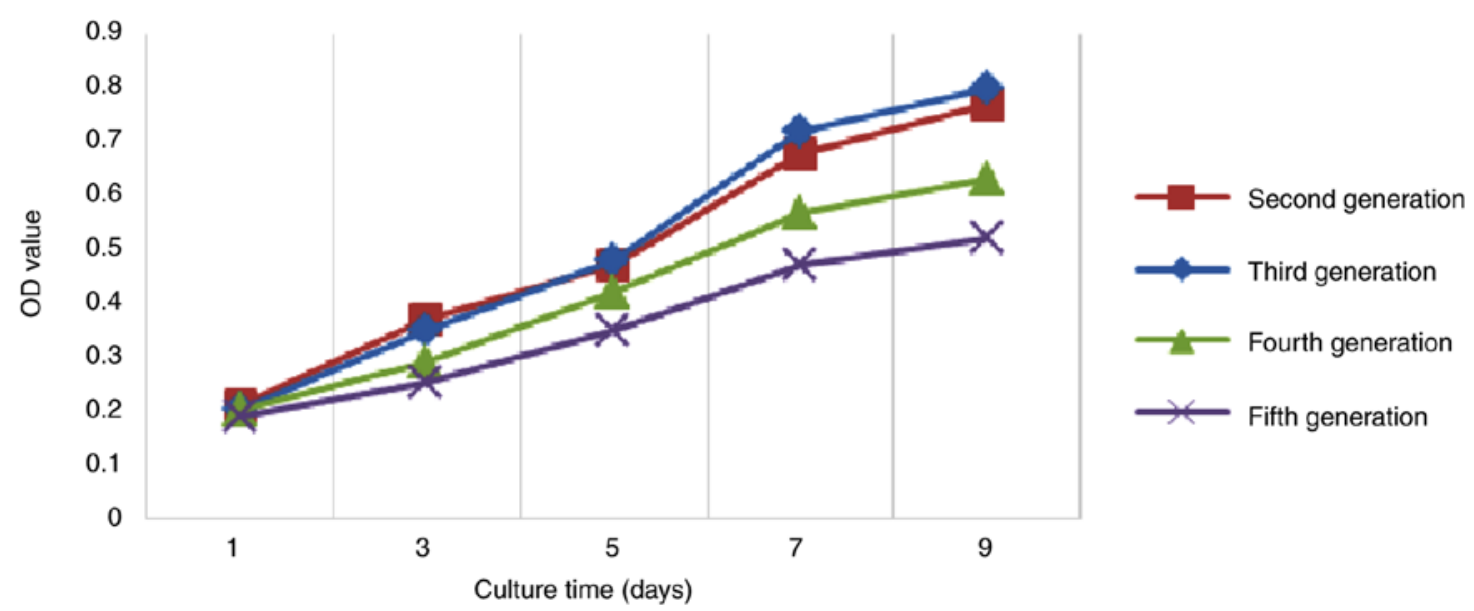

Figure 3. Analysis of proliferative activity of GMSCs. The MTT assay results demonstrated that GMSCs exhibited logarithmic growth on days 3-4 following inoculation, and the cell number tended to stabilize on days 7-8. The third-generation cells exhibited strong proliferative capacity. GMSCs, gingiva-derived mesenchymal stem cells; OD, optical density.

centrifugation with a specialized centrifugation procedure, CGF generation is characterized by three phases: i) A superior phase represented by the serum; ii) an interim phase represented by a very large and dense polymerized fibrin clot with aggregated platelets and CGF; and iii) a dense, viscous lower red portion consisting of coagulated red blood cells (Fig. 4A). As shown in Fig. 4B, CGF gels appeared with a light yellow gelatinous, translucent, soft, elastic, smooth surface.

The CCK- 8 assay results indicated that the optical density value increased gradually during the experimental period. Compared with the control group, $10 \%$ CGF significantly promoted the proliferation of GMSCs $(\mathrm{P}<0.05$; Fig. 4C). However, at concentrations $>10 \%$, the proliferative activity gradually decreased, although it remained higher compared with the control group (Fig. 4C).

CGF promote osteogenic differentiation of GMSCs. ALP is an exoenzyme of osteoblasts and its expression is an obvious marker of osteoblast differentiation. Through analyzing the activity of ALP in the three groups, it was demonstrated that the ALP activity in the experimental group was highest at 7, 14 and 24 days. Furthermore, significant differences were observed on pairwise analysis between groups $(\mathrm{P}<0.01$ vs control group; $\mathrm{P}<0.05$ vs. pure mineralization group; Fig. $5 \mathrm{~A}$ ). These findings suggest that CGF can induce osteogenic differentiation of GMSCs.

To further verify that CGF can induce osteogenic differentiation of GMSCs, Alizarin Red S staining was performed at 7, 14 and 24 days, and the results indicated that the experimental and the pure mineralization groups exhibited mineralized nodules. With the extension of experimental time, the staining area and density were gradually increased, and a large number of nodules were observed. The mineralized nodules were the most prevalent and the osteogenic induction was most prominent on the 21 st day. Through comparative analysis, it was demonstrated that the mineralized nodules in the experimental group with the addition of CGF appeared earlier and with a higher prevalence and density (Fig. 5B). This further supports the previous finding that CGF can induce osteogenic differentiation of GMSCs.
$C G F$ promote the $m R N A$ expression of DMP1, DSPP, BMP2 and RUNX2. In order to elucidate whether DMP1, DSPP, BMP2 and RUNX2 are involved in the CGF-induced differentiation of GMSCs, RT-qPCR was used to detect their mRNA expression. The results indicated that the mRNA expression of these four genes was significantly higher in the experimental group compared with that in the control group $(\mathrm{P}<0.01$; Fig. 6). Therefore, it was hypothesized that DMP1, DSPP, BMP2 and RUNX2 are involved in the CGF-induced mineralization of GMSCs.

$C G F$ promote the protein expression of DMP1, DSPP, BMP2 and RUNX2. In order to further elucidate the mechanism of action of CGF, the protein expression of DMP1, DSPP, BMP2 and RUNX2 was measured by western blotting, and the results indicated that the protein expression was significantly increased compared with the control group $(\mathrm{P}<0.01$; Fig. 7A and B). This finding further confirmed that CGF can promote the osteogenic differentiation of GMSCs.

\section{Discussion}

MSCs play an important role in tissue engineering and immunotherapy due to their multidirectional differentiation potential and ability to regulate immune responses. At present, a variety of MSCs have been identified in the oral cavity, including dental pulp stem cells, dental follicle stem cells, stem cells from the apical papilla, stem cells from human exfoliated deciduous teeth, periodontal vascular stem cells and GMSCs (15). It was previously reported that GMSCs display characteristics similar to those of stem cells and have a multidirectional differentiation potential. Therefore, the gingiva may be the source of novel seed cells $(16,17)$. Previous studies have also demonstrated that GMSCs have the ability to differentiate into bone, cartilage and adipose tissue, as well as endothelial-like cells and smooth muscle-like cells, under the influence of different cytokines (18-21).

Compared with BMSCs, GMSCs are easy to isolate and homogenize, they proliferate quickly and exhibit a stable morphology following multiple generations (4). In the present 
A

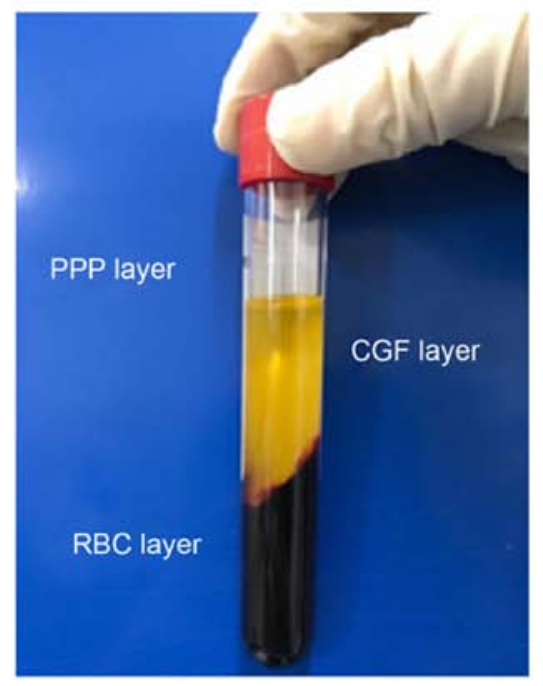

B

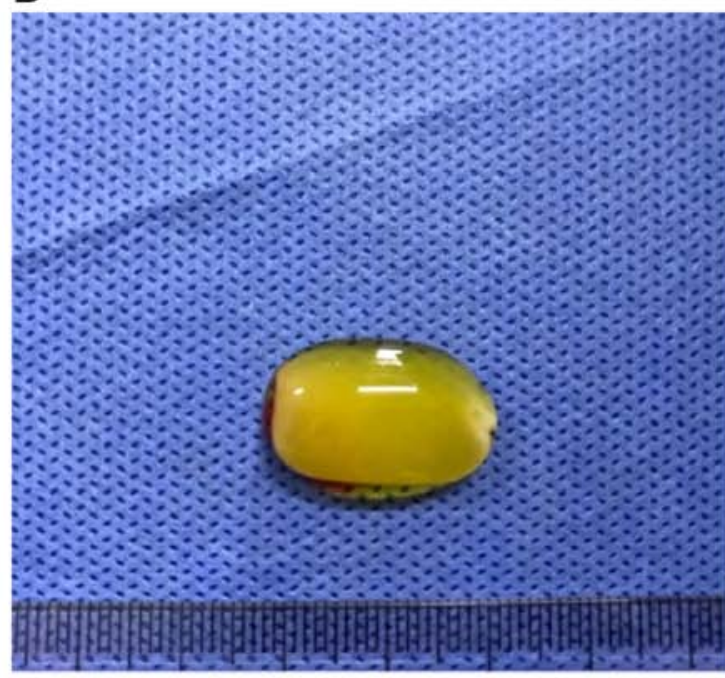

C

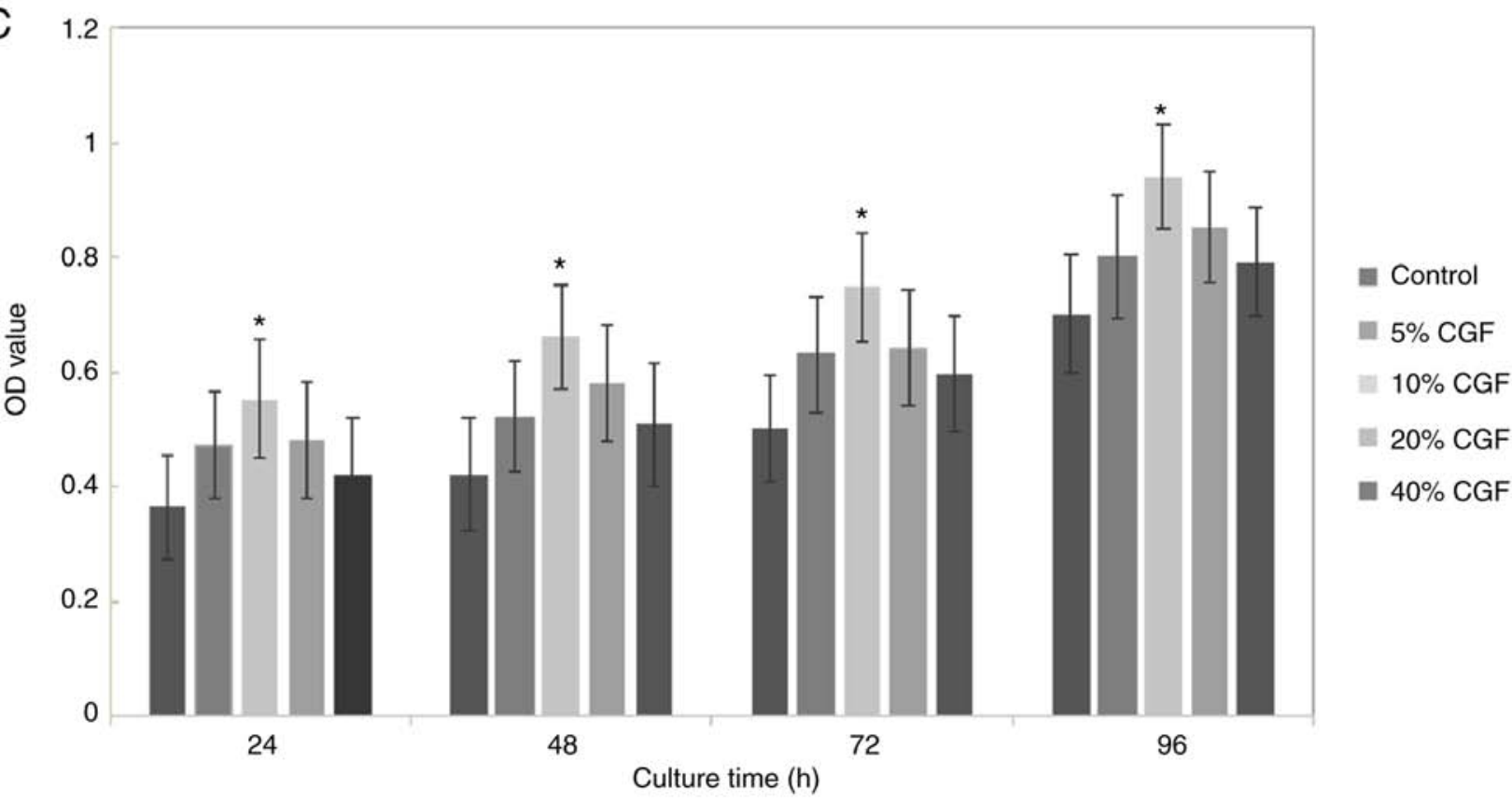

Figure 4. CGF. (A) The following blood fractions were obtained by centrifugation: i) A superior phase represented by the serum (platelet-poor plasma; PPP); ii) an interim phase represented by a large and dense polymerized fibrin clot containing CGF, white blood cells and stem cells; and iii) the lower red blood cell (RBC) layer. (B) The CGF gel exhibited a light yellow gelatinous, translucent, soft, elastic, smooth surface. (C) The Cell Counting Kit- 8 assay results demonstrated that the optical density (OD) value increased gradually over time. Compared with the control group, $10 \% \mathrm{CGF}$ significantly promoted the proliferation of GMSCs. "P<0.05. CGF, concentrated growth factors; GMSCs, gingiva-derived mesenchymal stem cells.

study, samples were collected from the normal gingival tissue of patients undergoing crown lengthening surgery. Through flow cytometry, immunohistochemical staining and immunofluorescence staining, the expanded culture cells were identified. The results of flow cytometry demonstrated that the positive rates of CD73, CD90, CD105 and CD45 were $99.83,99.74,82.35$ and $2.05 \%$, respectively, suggesting that the cells cultured in the present study displayed the biological characteristics of MSCs. To verify this result, immunohistochemical and immunofluorescence staining was performed. Immunohistochemical staining revealed positive expression of vimentin, CD73 and CD90, and negative expression of cytokeratin. Immunofluorescence staining revealed positive expression of CD90, S100A4 and vimentin, and negative expression of CK. Taken together, these findings indicated that the cultured cells in the present study were GMSCs.

CGF represent a novel generation of platelet concentrate (22). Due to the advanced extraction technology and specialized equipment, the preparation process is simple and does not require any synthetic or catalytic substances. CGF derived from the patient's own venous blood exclude potential cross-infection, toxicity and immunogenicity; thus, the application is highly safe (23). As CGF are derived from autologous venous blood, the growth factor levels are higher. Previous studies have demonstrated that CGF can promote bone formation and differentiation $(24,25)$. It has also been reported that CGF can improve and enhance tissue repair and regeneration $(7,11,12)$. 


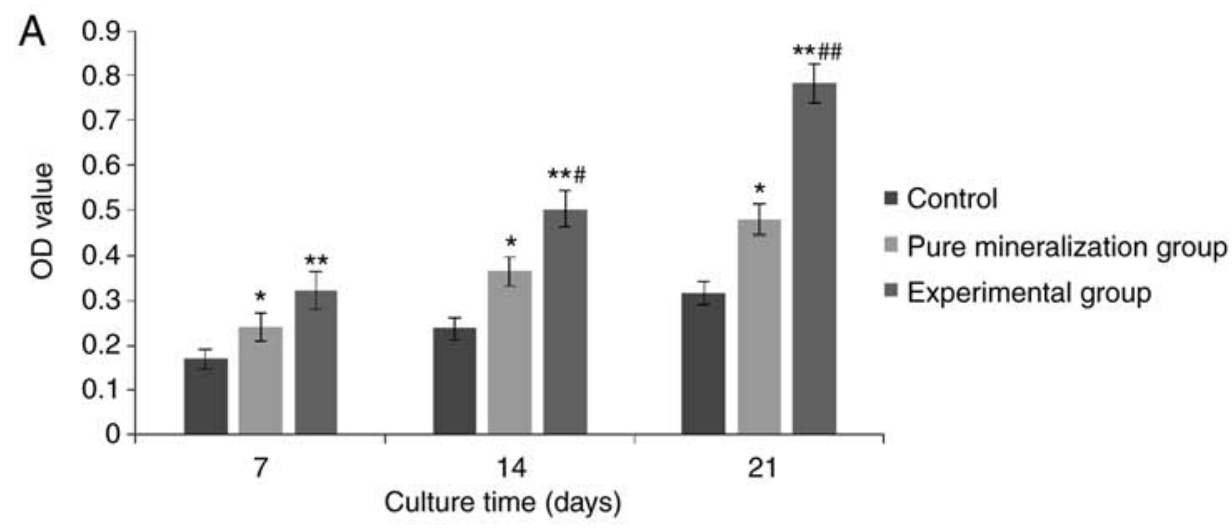

B
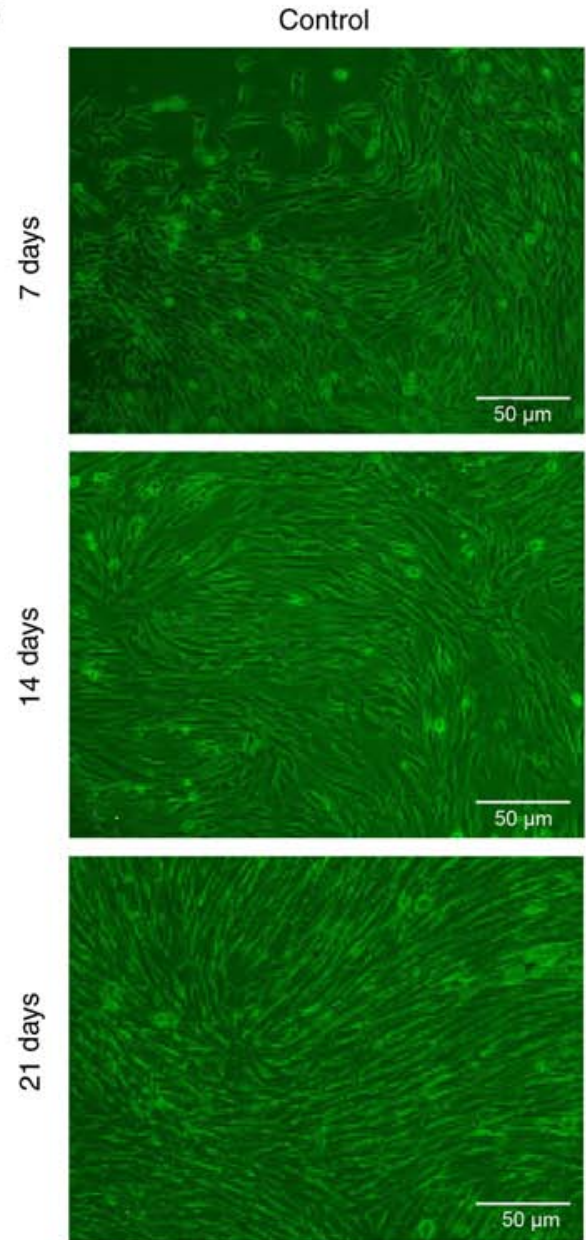

Pure mineralization group
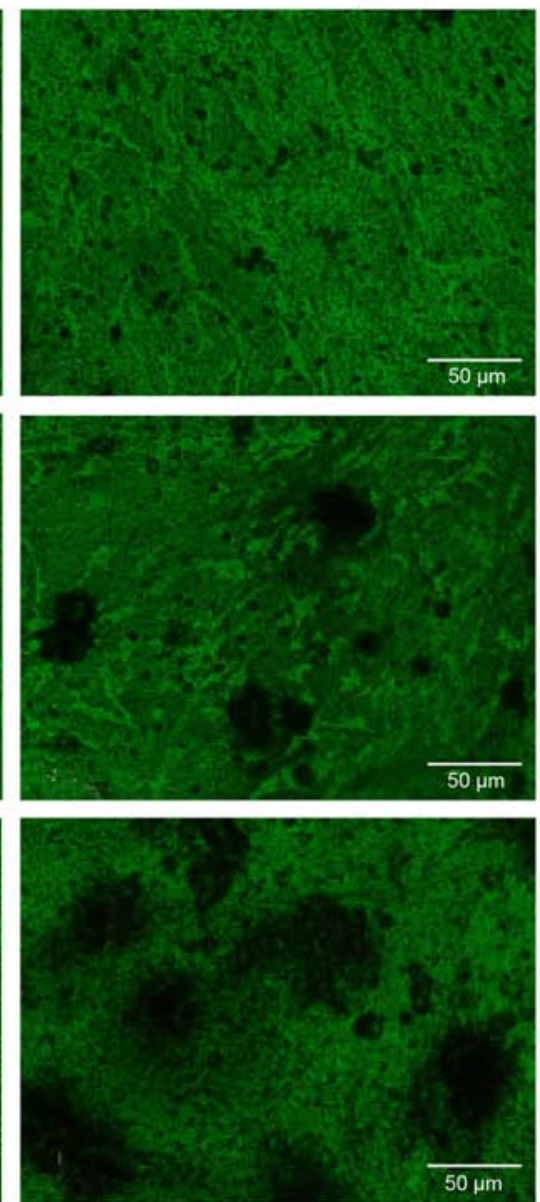

Experimental group
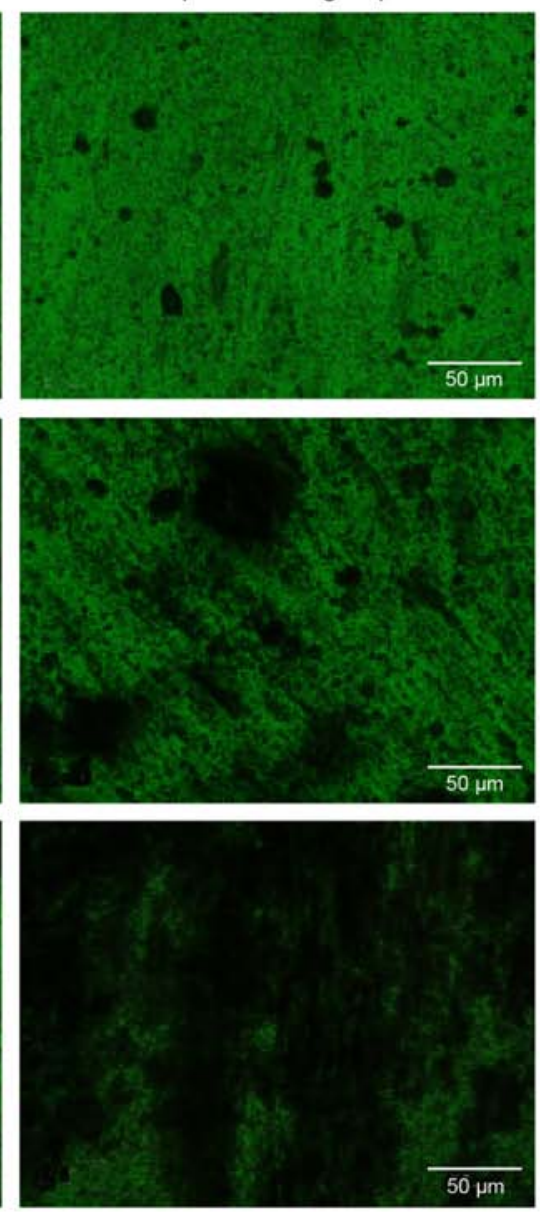

Figure 5. Effect of CGF on GMSC osteogenic differentiation at different time points. (A) Effect of CGF on alkaline phosphatase activity in GMSCs at different time points. Data are presented as the mean \pm standard deviation. ${ }^{*} \mathrm{P}<0.05$ indicated pure mineralization group vs. control group; ${ }^{* *} \mathrm{P}<0.01$ indicated experimental group vs. control group; ${ }^{\#} \mathrm{P}<0.05$ indicated experimental group at 14 days vs. experimental group at 7 days; ${ }^{\# \#} \mathrm{P}<0.01$ indicated experimental group at 21 days vs. experimental group at 7 days. (B) Mineralized nodule formation analysis. The Alizarin Red S staining results indicated that mineralized nodules appeared in the experimental group earlier, and at a higher number and density. Osteogenesis was most marked on the 21st day after mineralization induction (magnification, x200). CGF, concentrated growth factors; GMSCs, gingiva-derived mesenchymal stem cells.

In order to elucidate whether CGF also affect the proliferation and differentiation of GMSCs, a preliminary analysis of cell activity was subsequently conducted by adding CGF extract in vitro. During the experiment, five different CGF concentration gradients were used. The CCK- 8 results demonstrated that CGF enhanced the proliferation of GMSCs within the range of $10 \%$ concentration. However, when the concentration was $>10 \%$, a decrease in proliferative activity was observed, although it remained higher compared with the control group. Based on the analysis detailed above, it was demonstrated that CGF can promote the proliferation of GMSCs cells at an optimal concentration of $10 \%$. The effect of different concentrations of CGF on the proliferation capacity of GMSCs was also analyzed through a literature review. The proliferative effect of CGF is not only attributed to the various growth factors, but also to the three-dimensional fibrin network structure of platelets, white blood cells and growth factors (9). Platelets and various growth factors bond with fibrin mole- 

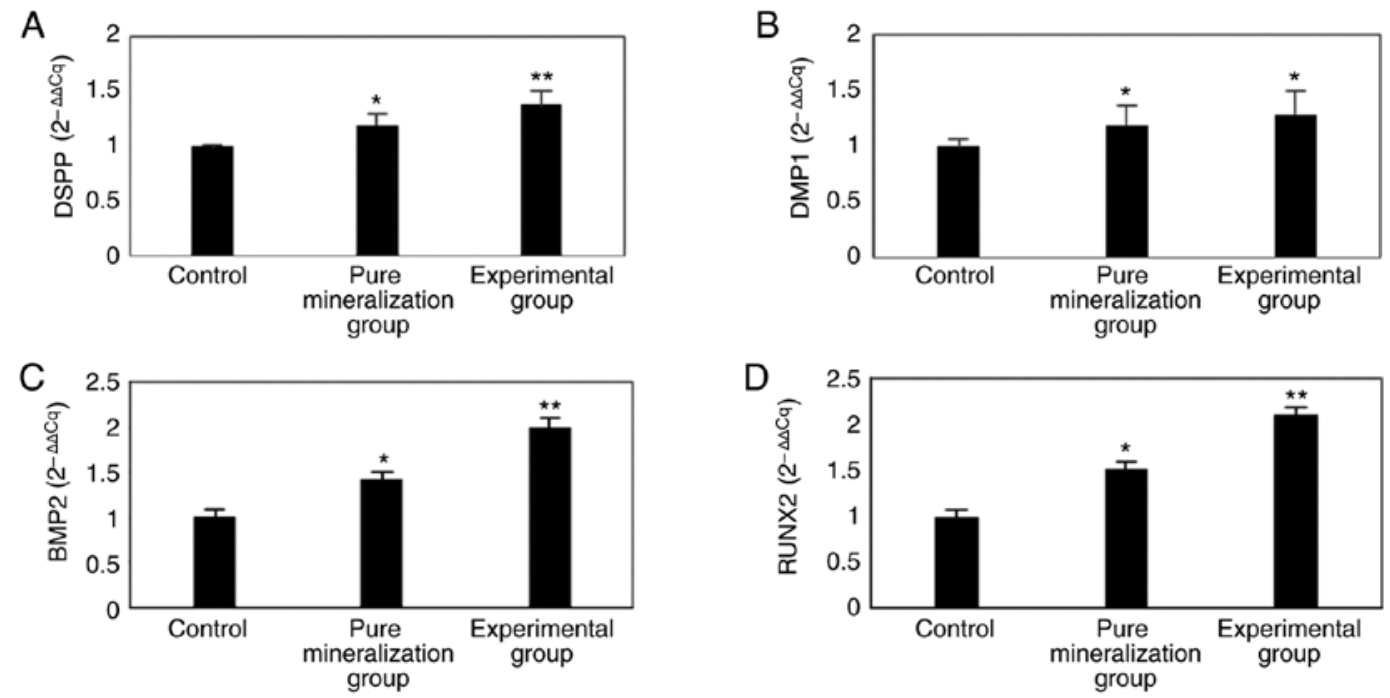

Figure 6. mRNA expression of DSPP, DMP1, BMP2 and RUNX2 on the 21st day after mineralization induction. The RT-qPCR results indicated that the (A) DSPP, (B) DMP1, (C) BMP2 and (D) RUNX2 mRNA expression were significantly increased. "P $<0.05$ indicated pure mineralization group vs. control group ${ }^{* * *} \mathrm{P}<0.01$ indicated experimental group vs. control group. DSPP, dentin sialophosphoprotein; DMP1, dentin matrix acidic phosphoprotein 1; BMP2, bone morphogenetic protein 2; RUNX2, runt-related transcription factor 2; RT-qPCR, reverse transcription-quantitative polymerase chain reaction.
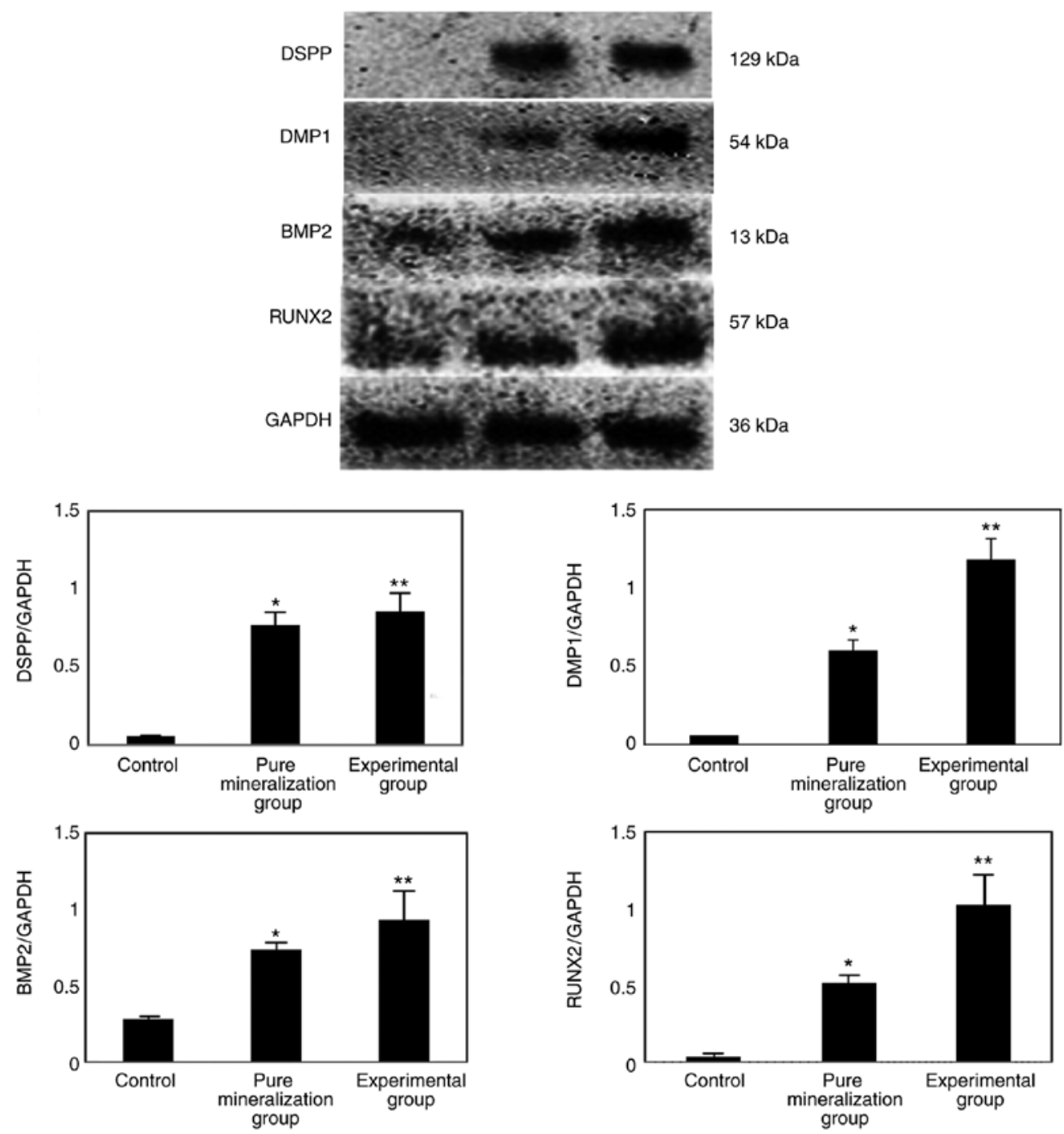

Figure 7. Protein expression of DSPP, DMP1, RUNX2 and BMP2 on the 21st day after mineralization induction. The western blotting results indicated that the DSPP, DMP1, BMP2 and RUNX2 protein expression was significantly increased. ${ }^{*} \mathrm{P}<0.05$ indicated pure mineralization group vs. control group; ${ }^{* *} \mathrm{P}<0.01$ indicated experimental group vs. control group. DSPP, dentin sialophosphoprotein; DMP1, dentin matrix acidic phosphoprotein 1; BMP2, bone morphogenetic protein 2; RUNX2, runt-related transcription factor 2. 
cules, which facilitate the adhesion of cell components and create an appropriate microenvironment for cell migration. However, the inability of high CGF concentration to promote gingival stem cell proliferation may be associated with the $\mathrm{pH}$ value in the cell environment. It has previously been proposed that $\mathrm{pH}$ value is affected by the change in platelet count, which may negatively affect cell proliferation (26).

ALP, as a marker of osteoblast differentiation, first appears in the early stages of cell mineralization induction and may be used as an early osteogenesis marker $(27,28)$. As the induction time increases and the osteogenic differentiation of cells progresses, its activity gradually increases. Therefore, the osteogenic function of the GMSCs can be evaluated. In order to explore the ability of CGF to induce osteogenic differentiation of GMSCs, ALP activity was detected. The results indicated that the ALP activity of the experimental group was highest at 7,14 and 21 days, suggesting that CGF can promote the osteogenesis of GMSCs. Changing calcium salt levels is a marker of bone cell proliferation and differentiation, and of the osteogenic potential of bone tissue. Therefore, the ability of osteoblasts to differentiate may be identified by determining the calcium salt deposition in the cell matrix of each group by Alizarin Red S staining. Through comparison of the experimental data among the three groups, it was demonstrated that the mineralized nodules in the experimental group with added CGF appeared earlier, and the number and density of the nodules were higher compared with the other two groups. Mineralization images revealed that the mineralized nodules were the most prevalent and the osteogenic induction was the most marked on day 21. This further verified that CGF can promote the osteoblastic differentiation of GMSCs.

DSPP is an extracellular matrix protein closely associated with tooth development, which has been demonstrated to play a central role in the formation and growth of hydroxyapatite crystals in the extracellular matrix of hard tissues, such as bones and teeth (29). DMP1 is a type of hyperphosphorylated hyperacidic non-collagen, which is mainly expressed in mineralized tissues, including bones and teeth. Due to its acidic domain and negative charge, it strongly binds with calcium ions and promotes the formation of hydroxyapatite (30-32). RUNX2 is associated with transcription during bone and tooth development, participates in dental crown formation and promotes odontoblast differentiation $(33,34)$. BMP2 promotes the self-renewal of stem cells, promotes the differentiation of mesenchymal cells into bone and cartilage, and participates in the development of various organs $(35,36)$. In order to investigate whether these four genes are involved in the induction of osteogenic differentiation of GMSCs by CGF, RT-qPCR and western blot analyses were conducted to quantify RNA and protein expression of the experimental, pure mineralization and control groups on the 21st day after mineralization induction. The RT-qPCR results indicated that DSPP, DMP1, BMP2 and RUNX2 were significantly upregulated compared with the control group. This finding indicates that CGF likely promotes osteoblastic differentiation of GMSCs cells by regulating the expression of DSPP, DMP1, BMP2 and RUNX2. The western blotting results were consistent with the RT-qPCR results, further supporting the hypothesis that CGF upregulates DSPP, DMP1, BMP2 and RUNX2 to promote the proliferation and osteogenic differentiation of GMSCs.
In conclusion, the findings of the present study revealed that CGF can significantly promote the proliferation and osteogenic differentiation of GMSCs. Therefore, CGF appears to be promising for certain applications in tissue engineering for tooth regeneration and repair. However, the mechanisms underlying the regulation of the DSPP, DMP1, BMP2 and RUNX2 signaling pathways by CGF remain to be fully elucidated.

\section{Acknowledgements}

Not applicable.

\section{Funding}

The present study was supported by grants from the Medical Science Project of Sichuan Province (no. S17073) and the University-level Scientific Research Project of Southwest Medical University (no. 2017-ZRQN-083) and the General Program of Sichuan Provincial Science and Technology Department Applied Basic Research (no. 2018JY040).

\section{Availability of data and materials}

The datasets generated and/or analyzed during the present study are available from the corresponding author on reasonable request.

\section{Authors' contributions}

XL conceived of the study, provided materials and samples, and participated in data collection, analysis and interpretation of the results. MN provided administrative support. XC, YC and $\mathrm{MZ}$ provided materials and samples. $\mathrm{YC}$ and $\mathrm{YH}$ contributed to data collection. XC and PS contributed to interpretation of the results. All authors have read and approved the final version of this manuscript for publication.

\section{Ethics approval and consent to participate}

The study was approved by the Ethics Committee of the Affiliated Stomatological Hospital of Southwest Medical University and all the patients included in the study signed an informed consent prior to tissue collection. All procedures were performed in accordance with the World Medical Association's Declaration of Helsinki.

\section{Patient consent for publication}

Not applicable.

\section{Competing interests}

All the authors declare that they have no competing interests to disclose.

\section{References}

1. Xu S, De Veirman K, De Becker A, Vanderkerken K and Van Riet I: Mesenchymal stem cells in multiple myeloma: A therapeutical tool or target? Leukemia 32: 1500-1514, 2018. 
2. Dominici M, Le Blanc K, Mueller I, Slaper-Cortenbach I, Marini F, Krause D, Deans R, Keating A, Prockop D and Horwitz E: Minimal criteria for defining multipotent mesenchymal stromal cells. The International Society for Cellular Therapy position statement. Cytotherapy 8: 315-317, 2006.

3. Stenderup K, Justesen J, Clausen C and Kassem M: Aging is associated with decreased maximal life span and accelerated senescence of bone marrow stromal cells. Bone 33: 919-926, 2003.

4. Tomar GB, Srivastava RK, Gupta N, Barhanpurkar AP, Pote ST, Jhaveri HM, Mishra GC and Wani MR: Human gingiva-derived mesenchymal stem cells are superior to bone marrow-derived mesenchymal stem cells for cell therapy in regenerative medicine. Biochem Biophys Res Commun 393: 377-383, 2010.

5. Mitrano TI, Grob MS, Carrión F, Nova-Lamperti E, Luz PA, Fierro FS, Quintero A, Chaparro A and Sanz A: Culture and characterization of mesenchymal stem cells from human gingival tissue. J Periodontol 81: 917-925, 2010.

6. Zhang Q, Shi S, Liu Y, Uyanne J, Shi Y, Shi S and Le AD: Mesenchymal stem cells derived from human gingiva are capable of immunomodulatory functions and ameliorate inflammation-related tissue destruction in experimental colitis. J Immunol 183: 7787-7798, 2009.

7. Kim TH, Kim SH, Sándor GK and Kim YD: Comparison of platelet-rich plasma (PRP), platelet-rich fibrin (PRF), and concentrated growth factor (CGF) in rabbit-skull defect healing. Arch Oral Biol 59: 550-558, 2014.

8. Qiao J and An N: Effect of concentrated growth factors on function and Wnt3a expression of human periodontal ligament cells in vitro. Platelets 28: 281-286, 2017.

9. Rodella LF, Favero G, Boninsegna R, Buffoli B, Labanca M, Scari G, Sacco L, Batani T and Rezzani R: Growth factors, CD34 positive cells, and fibrin network analysis in concentrated growth factors fraction. Microsc Res Tech 74: 772-777, 2011.

10. Tayapongsak P, O'Brien DA, Monteiro CB and Arceo-Diaz LY: Autologous fibrin adhesive in mandibular reconstruction with particulate cancellous bone and marrow. J Oral Maxillofac Surg 52: 161-165; discussion 166, 1994

11. Bozkurt Doğan Ş, Öngöz Dede F, Ballı U, Atalay EN and Durmuslar MC: Concentrated growth factor in the treatment of adjacent multiple gingival recessions: A split-mouth randomized clinical trial. J Clin Periodontol 42: 868-875, 2015.

12. Takeda Y, Katsutoshi K, Matsuzaka K and Inoue T: The effect of concentrated growth factor on rat bone marrow cells in vitro and on calvarial bone healing in vivo. Int J Oral Maxillofac Implants 30: 1187-1196, 2015.

13. Mosmann T: Rapid colorimetric assay for cellular growth and survival: Application to proliferation and cytotoxicity assays. J Immunol Methods 65: 55-63, 1983.

14. Livak KJ and Schmittgen TD: Analysis of relative gene expression data using real-time quantitative PCR and the 2(-Delta Delta C(T)) method. Methods 25: 402-408, 2001.

15. Heng BC, Zhang C, Deng X, Xiao Y, Pisciotta A, Kidwai F and Mitsiadis TA: Biomedical applications of dental and oral-derived stem cells. Stem Cells Int 2017: 2931054, 2017.

16. Ji J, Tong X, Huang X, Zhang J, Qin H and Hu Q: Patient-derived human induced pluripotent stem cells from gingival fibroblasts composited with defined nanohydroxyapatite/chitosan/gelatin porous scaffolds as potential bone graft substitutes. Stem Cells Transl Med 5: 95-105, 2016.

17. Fawzy El-Sayed KM and Dörfer CE: Gingival mesenchymal stem/progenitor cells: A unique tissue engineering gem. Stem Cells Int 2016: 7154327, 2016.

18. Liu X, Wang J, Dong F, Li H and Hou Y: Human gingival fibroblasts induced and differentiated into vascular endothelial-like cells. Dev Growth Differ 58: 702-713, 2016.

19. Liu X, Wang J, Dong F, Li H and Hou Y: Induced differentiation of human gingival fibroblasts into VSMC-like cells. Differentiation 95: 1-9, 2017

20. Xu X, Chen C, Akiyama K, Chai Y, Le AD, Wang Z and Shi S Gingivae contain neural-crest- and mesoderm-derived mesenchymal stem cells. J Dent Res 92: 825-832, 2013.
21. Ansari S, Diniz IM, Chen C, Sarrion P, Tamayol A, Wu BM and Moshaverinia A: Human periodontal ligament- and gingiva-derived mesenchymal stem cells promote nerve regeneration when encapsulated in alginate/Hyaluronic acid 3D scaffold. Adv Healthc Mater 6, 2017.

22. Bernardi S, Mummolo S, Tecco S, Continenza MA and Marzo G: Histological characterization of Sacco's concentrated growth factors membrane. Int J Morphol 35: 114-119, 2017.

23. Qin J, Wang L, Sun Y, Sun X, Wen C, Shahmoradi M and Zhou Y: Concentrated growth factor increases Schwann cell proliferation and neurotrophic factor secretion and promotes functional nerve recovery in vivo. Int J Mol Med 37: 493-500, 2016.

24. Kim JM, Sohn DS, Bae MS, Moon JW, Lee JH and Park IS: Flapless transcrestal sinus augmentation using hydrodynamic piezoelectric internal sinus elevation with autologous concentrated growth factors alone. Implant Dent 23: 168-174, 2014.

25. Sohn DS, Heo JU, Kwak DH, Kim DE, Kim JM, Moon JW, Lee JH and Park IS: Bone regeneration in the maxillary sinus using an autologous fibrin-rich block with concentrated growth factors alone. Implant Dent 20: 389-395, 2011.

26. Liu Y, Kalén A, Risto O and Wahlström O: Fibroblast proliferation due to exposure to a platelet concentrate in vitro is $\mathrm{pH}$ dependent. Wound Repair Regen 10: 336-340, 2002.

27. Mornet E, Stura E, Lia-Baldini AS, Stigbrand T, Ménez A and Le Du MH: Structural evidence for a functional role of human tissue nonspecific alkaline phosphatase in bone mineralization. J Biol Chem 276: 31171-31178, 2001.

28. Douglas TE, Messersmith PB, Chasan S, Mikos AG, de Mulder EL, Dickson G, Schaubroeck D, Balcaen L, Vanhaecke F, Dubruel P, et al: Enzymatic mineralization of hydrogels for bone tissue engineering by incorporation of alkaline phosphatase. Macromol Biosci 12: 1077-1089, 2012.

29. Choi YS, Lee JY, Suh JS, Lee G, Chung CP and Park YJ: The mineralization inducing peptide derived from dentin sialophosphoprotein for bone regeneration. J Biomed Mater Res A 101: 590-598, 2013

30. Gericke A, Qin C, Sun Y, Redfern R, Redfern D, Fujimoto Y, Taleb H, Butler WT and Boskey AL: Different forms of DMP1 play distinct roles in mineralization. J Dent Res 89: 355-359, 2010.

31. Bhatia A, Albazzaz M, Espinoza Orias AA, Inoue N, Miller LM, Acerbo A, George A and Sumner DR: Overexpression of DMP1 accelerates mineralization and alters cortical bone biomechanical properties in vivo. J Mech Behav Biomed Mater 5: 1-8, 2012.

32. Narayanan K, Srinivas R, Ramachandran A, Hao J, Quinn B and George A: Differentiation of embryonic mesenchymal cells to odontoblast-like cells by overexpression of dentin matrix protein 1. Proc Natl Acad Sci USA 98: 4516-4521, 2001.

33. Monteiro N, Ribeiro D, Martins A, Faria S, Fonseca NA, Moreira JN, Reis RL and Neves NM: Instructive nanofibrous scaffold comprising runt-related transcription factor 2 gene delivery for bone tissue engineering. ACS Nano 8: 8082-8094, 2014.

34. Li S, Kong H, Yao N, Yu Q, Wang P, Lin Y, Wang J, Kuang R, Zhao X, Xu J, et al: The role of runt-related transcription factor 2 (Runx2) in the late stage of odontoblast differentiation and dentin formation. Biochem Biophys Res Commun 410: 698-704, 2011.

35. Zhang C, Meng C, Guan D and Ma F: BMP2 and VEGF165 transfection to bone marrow stromal stem cells regulate osteogenic potential in vitro. Medicine (Baltimore) 97: e9787, 2018

36. Qin W, Yang F, Deng R, Li D, Song Z, Tian Y, Wang R, Ling $J$ and Lin $Z$ : Smad 1/5 is involved in bone morphogenetic protein-2-induced odontoblastic differentiation in human dental pulp cells. J Endod 38: 66-71, 2012.

This work is licensed under a Creative Commons Attribution-NonCommercial-NoDerivatives 4.0 International (CC BY-NC-ND 4.0) License. 